\title{
Relationship between the response to allergen, histamine and hypertonic saline nasal challenge and subjective reactivity to irritants in subjects with seasonal allergic rhinitis
}

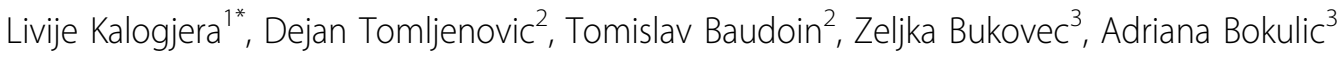 \\ From The 10th Symposium of Experimental Rhinology and Immunology of the Nose (SERIN 2015) \\ Stockholm, Sweden. 19-21 February 2015
}

\section{Background}

Patients with seasonal allergic rhinitis (SAR) may have symptoms triggered by environmental changes and irritants exposure in the absence of allergen. It is hypothesized that level of hyperreactivity in mixed rhinitis is related the inflammation in the late phase response, stimulation of sensory nerves and transient receptor potential (TRP) channels. The study was done to compare subjective and objective response to the low-dose allergen, histamine and hypertonic saline challenge, with subjective responsiveness to common irritants.

\section{Methods}

A group of 45 non-asthmatic patients with SAR, were submitted to consecutive nasal provocations, out of season, with 1.000 I.U. of allergen, $80 \mathrm{mcg}$ of histamine 24 hours after allergen challenge, and 48 hours after histamine with $2 \%$ hypertonic saline (HTS). Prior to the challenges patients filled in a modified Cinncinati irritant index questionnaire testing subjective sensitivity to 20 common environmental triggers (scale 0-10). Before and after challenges visual analog scale (VAS) subjective scores of nasal and ocular symptoms, and nasal lavages were done 15 minutes, 4 and 24 hours after allergen, and 15 minutes after histamine and HTS. Nasal lavages were analysed for eosinophil cation protein (ECP) 4 and 24 hours after allergen and substance P (SP) 15 minutes after HTS challenge.

\section{Results}

Highest score for individual irritant in the Cinncinati irritant index scale (CIS) was found for cold air and

${ }^{1}$ University Hospital Centre Zagreb School of Medicine, Zagreb, Croatia Full list of author information is available at the end of the article tobacco smoke, followed by weather changes and cleaning solutions. Total score in CIS correlated significantly with differences in VAS prior to and after allergen challenge for burning sensation in the nose (rho 0.47, $\mathrm{p}=0.001$ ) and eye itch (rho 0.49, $\mathrm{p}=0.0001$ ). On the other hand, subjective responsiveness for cold air correlated with VAS difference for obstruction (rho 0.32, $\mathrm{p}=0.03$ ), secretion (rho $0.33, \mathrm{p}=0.03)$ and nose itch $(0.38, \mathrm{p}=0.008)$. There was no correlation between ECP and SP levels in lavages and sneezing count with subjective responsiveness to irritants (total CIS score).

\section{Conclusions}

Different patterns of symptoms after low-dose nasal challenges in patients with mixed rhinitis may be attributed to sensitivity to specific set of irritants. Severity of inflammation after such challenges does not reflect subjective irritant sensitivity. In patients with high subjective sensitivity to a broad panel of irritants, a psychosomatic component of multiple chemical sensitivity may be considered.

\section{Authors' details \\ ${ }^{1}$ University Hospital Centre Zagreb School of Medicine, Zagreb, Croatia. ${ }^{2}$ University Hospital Centre Zagreb School of Medicine, ENT/HNS Dept, Zagreb, Croatia. ${ }^{3}$ University Hospital Centre Zagreb School of Medicine, Endo Lab, Zagreb, Croatia.}

Published: 26 June 2015
doi:10.1186/2045-7022-5-S4-O10
Cite this article as: Kalogjera et al.: Relationship between the response
to allergen, histamine and hypertonic saline nasal challenge and
subjective reactivity to irritants in subjects with seasonal allergic rhinitis.
Clinical and Translational Allergy 2015 5(Suppl 4):O10. 